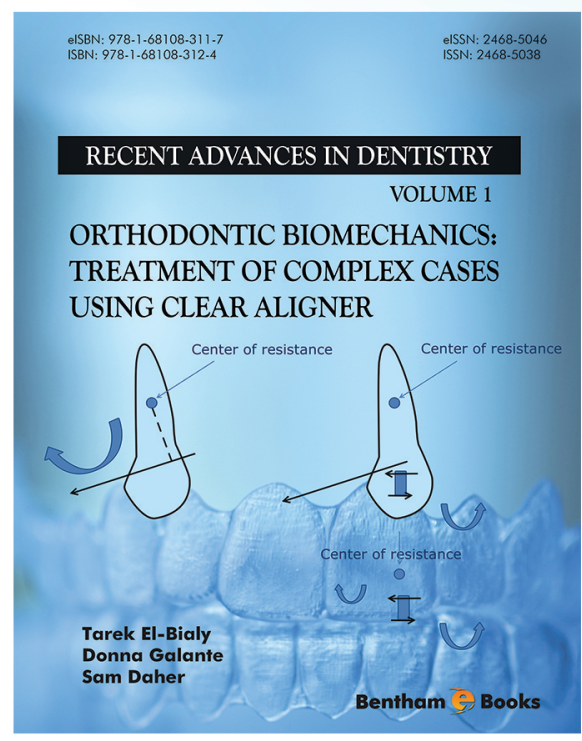

\title{
Authors:
}

Tarek El-Bialy

Donna Galante

Sam Daher

eISBN: $978-1-68108-311-7$

\section{Orthodontic Biomechanics: Treatment Of Complex Cases Using Clear Aligner}

\section{Wur.ehooks.henthamseience.com/hook/9781681083117/}

\section{About the eBook}

Orthodontic Biomechanics describes the mechanics behind the treatment of complex orthodontic cases using clear aligners. The volume explains a variety of complex malocclusions including increased teeth crowding, spacing, overjet, overbite, open bite, major jaw discrepancies, underbite and much more

\section{Contents}

- Introduction/History of Clear Aligners

- Science and Practice of Clear Aligners

- Orthodontic Diagnosis and Treatment Planning

- Unique Features of Clear Aligners Compared to Regular Orthodontic Appliances

- Orthodontic Biomechanics Using Clear Aligners

- Moderate and Severe Crowding Class I Cases

- Treatment of Class II Malocclusion Using Clear Aligners

- Treatment of Class III Malocclusion Using Clear Aligners

- Treatment of Anterior Open Bite Using Clear Aligner Therapy

- Treatment of Facial Asymmetry Using Clear Aligners

- Treatment of Challenging Cases Using Clear Aligners

- Surgical Orthodontics Treatment with Invisalign

- Future Directions with Clear Aligners

For Advertising Inquiries: Contact: marketing@benthamscience.org 